This item was submitted to Loughborough's Research Repository by the author.

Items in Figshare are protected by copyright, with all rights reserved, unless otherwise indicated.

\title{
Effective radiation field model to scattering - absorption applied in heterogeneous photocatalytic reactors
}

PLEASE CITE THE PUBLISHED VERSION

http://dx.doi.org/10.1016/j.cej.2015.05.056

\section{PUBLISHER}

(C) Elsevier

VERSION

AM (Accepted Manuscript)

\section{PUBLISHER STATEMENT}

This work is made available according to the conditions of the Creative Commons Attribution-NonCommercialNoDerivatives 4.0 International (CC BY-NC-ND 4.0) licence. Full details of this licence are available at: https://creativecommons.org/licenses/by-nc-nd/4.0/

\section{LICENCE}

CC BY-NC-ND 4.0

\section{REPOSITORY RECORD}

Mueses, Miguel Angel, Fiderman Machuca-Martinez, Aracely Hernandez-Ramirez, and Gianluca Li Puma. 2015. "Effective Radiation Field Model to Scattering - Absorption Applied in Heterogeneous Photocatalytic Reactors". figshare. https://hdl.handle.net/2134/19053. 


\title{
A NEW RADIATION SCATTERING - ABSORPTION MODEL APPLIED TO HETEROGENEOUS PHOTOCATALYTIC SOLAR REACTORS
}

\author{
Miguel Angel Mueses $^{(1)}$, Fiderman Machuca-Martinez ${ }^{(2)}$, Aracely Hernández-Ramirez $^{(3)}$ and \\ Gianluca Li Puma ${ }^{(4)}$ \\ ${ }^{(1)}$ Photocatalysis and Solar Photoreactors Engineering, Department of Chemical Engineering, \\ Universidad de Cartagena, A.A. 1382-Postal 195, Cartagena-Colombia \\ ${ }^{(2)}$ GAOX Research Group, School of Chemical Engineering, Universidad del Valle, A.A. 25360, \\ Cali-Colombia \\ (3) Universidad Autonóma de Nuevo León, Facultad de Ciencias Químicas, Cd. Universitaria, \\ C.P. 66450. San Nicola's de los Garza, NL, México \\ ${ }^{(4)}$ Environmental Nanocatalysis and Photoreaction Engineering, Department of Chemical \\ Engineering, Loughborough University, Loughborough LE11 3TU, United Kingdom \\ *Corresponding author:
}

\begin{abstract}
:
A new mathematical model for the calculation of the radiation field in heterogeneous photocatalytic reactors using the new concept of "Effective radiation field model" or ERFM is proposed. In this concept, the incident radiation associated to the photons flow is an energy cloud. The generated space-phase and the properties of the cloud are considered to be isotropic and independent of the propagation angle and photon frequency. The isotropic nature of the ERFM concept provides a simpler estimation of the radiation field of a photocatalyst suspension (particles and fluid) for polychromatic radiation spectra such as the solar spectrum.
\end{abstract}

The ERFM is an alternative for the calculus of the spatial distribution of the radiant energy in heterogeneous photocatalytic reactors as an extension of the concept associated to the overall volumetric rate photon absorption - OVRPA. The local volumetric rate of photon absorption (LVRPA) predicted by the ERFM were compared with the predictons from the Six Flux Model (SFM) and the rigorous solution of the radiative transfer equation (RTE).

The LVRPA calculated with the ERFM was found to be closer to the solution of the RTE by the Discrete Ordinate Method (DOM). This was attributed to the isotropic phase function which was adopted in both models (ERFM and RTE-DOM).

Keywords: radiation field, solar spectrum, radiant energy, LVRPA, solar photoreactors, photocatalyst.

\section{Abbreviations}


ERFM : $\quad$ Effective Radiation Field Model.

OVRPA : $\quad$ Overall Volumetric Rate Photon Absorption.

SFM: $\quad$ Six Flux Absorption-scaterring Model.

RTE: $\quad$ Radiative Transfer Equation.

DOM: $\quad$ Discrete Ordinate Method.

\section{Introduction}

Heterogeneous photocatalysis is a platform technology, for environmental, renewable energy and green synthesis applications. A great body of literature exists on the fundamental aspects of photocatalysis [1, 2] including the synthesis of photocatalytic materials, the mechanisms of photocatalytic oxidation of contaminants [3], the application of photocatalysis for environmental remediation [4], the production of renewable energy [5] and for the green synthesis of chemicals [6, 7]. However, less understood among the scientific community is the distribution of the radiation field in absorption-scattering media. Many published studies in photocatalysis neglect the effect of radiation absorption over the observed kinetics and in consequence the interpretation of the results may contains uncertainties. Such lack of knowledge may result from inherent complexities of the radiation-transport process in absorbing and scattering media [8].

The spatial distribution of radiation field and radiation absorption are key aspects in heterogeneous photocatalytic processes. In the presence of a radiation field made of photons of wavelength with energy higher than the band-gap of the semiconductor photocatalyst electronhole pairs can be generated, which in turn trigger reduction and oxidation reactions at the surface of the photocatalyst. Highly reactive radical species such as hydroxyl, peroxyl, radicals and superoxide anion, $[9,10]$ are often produced which can then oxidize or reduce organic and inorganic contaminants. There is consensus among the scientific community that the evaluation and optimization of the radiation absorption in solar photoreactors is a very important step, in order to realize a wider application of photocatalytic processes [2, 8, 11].

The direct and indirect relationship of the radiation field with the redox reaction kinetics is associated through the definition of the quantum yield of the photocatalytic reaction (the photon activation step of the semiconductor), and through the distribution of the local volumetric rate of photon absorption (LVRPA) [10-14]. Current efforts in mathematical modeling are centered in an adequate description of the LVRPA to facilitate the prediction of the experimental data on the heterogeneous photocatalytic degradation of contaminants in a fluid.

The LVRPA is conventionally modeled from the contribution of the specific radiation intensity (i.e, irradiance) $I_{\lambda}(\underline{\mathbf{x}}, \underline{\boldsymbol{\Omega}}, t)$ integrated over all propagation directions (Einstein $\cdot \mathrm{s}^{-1}$ ) and the global volumetric coefficient of absorption $k_{\lambda}\left(\mathrm{cm}^{-1}\right)$ [15]:

$e_{\lambda}^{a}=\kappa_{\lambda} \int_{\Omega} I_{\lambda}(\vec{x}, \vec{\Omega}) d \Omega d \lambda$

The value of $I_{\lambda}(\underline{\mathbf{x}}, \underline{\boldsymbol{\Omega}}, t)$ is obtained by solving the Radiative Transfer Equation (RTE), which describes photon transport through an immobilized material or though a material dispersed in a fluid. At steady state, the primary form of the RTE used to model the radiation fields in heterogeneous photocatalytic reactors is [15]: 


$$
\frac{d}{d s} I_{\lambda}(\vec{x}, \vec{\Omega})=-\left[\kappa_{\lambda}(\vec{x})+\sigma_{\lambda}(\vec{x})\right] I_{\lambda}(\vec{x}, \bar{\Omega})+\frac{1}{4 \pi} \sigma_{\lambda}(\vec{x}) \int_{\overrightarrow{\Omega^{\prime}=4 \pi}} \rho\left(\lambda^{\prime} \rightarrow \lambda, \Omega^{\prime} \rightarrow \Omega\right) I_{\lambda}(\vec{x}, \vec{\Omega}) d \bar{\Omega}^{\prime}
$$

where $I_{\lambda}$ is the incident radiation at wavelength $\lambda, s$ is the generalized coordinate, $\kappa_{\lambda}$ is the absorption volumetric coefficient $\left(\mathrm{m}^{-1}\right), \sigma_{\lambda}$ is the scattering volumetric coefficient $\left(\mathrm{m}^{-1}\right), \vec{x}$ represents the coordinate vector, and $\rho\left(\lambda^{\prime} \rightarrow \lambda, \Omega^{\prime} \rightarrow \Omega\right)$ is the scattering phase function (the term in parentheses represents the frequency re-directing in radiation $\lambda$ and propagation $\Omega$ [16]):

In general, the modeling of the radiation field in photocatalytic reactors has been applied on systems using artificial radiation sources (UV lamps). The main modeling approach follows the rigorous solution of the RTE (Eq. 2) using the Discrete Ordinate Method (DOM) proposed by Duderstadt and Martin $[10,15]$. This method has been applied to plane photoreactor geometries [11, 15, 17], tubular photoreactors [10, 13, 18, 19], and compound parabolic collectors (CPC) reactors [11, 12, 20-22] using monochromatic and polychromatic irradiation sources and at steady state.

The RTE solved by the DOM method allow an accurate prediction of the radiation field in heterogeneous photocatalytic systems. This approach has been validated with simulation data based on the solution of Mie theoretical equation. Among different expressions of the scattering phase function, it has been found that the isotropic phase function is one of the most appropriate to describe the scattering properties of semiconductor photocatalysts [14, 23]. However, since the RTE is an integral-differential equation the application of the DOM for its solution requires a high degree of numerical accuracy, which is reflected in a high demand in computational time [12, 13, 15, 23]. Additionally, boundary conditions need to be evaluated with accurate and specialized actinometric techniques [24].

These limitations are a disadvantage for the simulation and scaling-up of photocatalytic reactors at the solar scale. Furthermore, diurnal fluctuations of the solar energy do not permit to have a steady incident radiation flux and atmospheric effects and geographical conditions further increase the complexity of the model solutions and actinometric treatment [26-31]. Under a variable solar flux the computational demand of the RTE-DOM becomes quite prohibitive.

With regards to solar photocatalytic reactors, the Six-Flux Scattering-Absorption Model (SFM) has been implemented to describe radiation fields and rate of photon absorption in contaminant degradation applications [25, 32-33]. This method was first proposed as a modification of the Two-Flux Model (TFM) [34, 35], and was validated through a comparison with the Monte Carlo simulation of the radiation field in a photoreactor with plane geometry [36].

The SFM in its original form describes photon scattering as a diffuse function (although this is not a limiting condition) and considers that incident photons have the probability to disperse in trajectories described by the six directions of the Cartesian coordinates (hence the name) [25, 26]. Its mathematical structure is of algebraic nature, therefore, its implementation in reactors to different scales and radiation sources is practical, with a low complexity in numeric procedures and short computational times [36]. Nevertheless, the presence of a diffuse phase function may cause the method to fail in correctly predict radiant field performances generated by simulation through the solution of Mie theoretical equation [9].

Other approaches to the modeling of radiant fields in heterogeneous photocatalytic reactors deal with stochastic models, which are based on Monte Carlo simulation [37-39], or with the application of Computational Fluid Dynamics (CFD) [21, 40-43]. The latter based on the constitutive equations of continuous-medium mechanics. Although these models are as accurate as the DOM solution, they have not been currently implemented at a solar scale due to their 
high computational and mathematical cost, which is not practical with fluctuating solar fluxes.

In this study, a new model for the evaluation of the radiation field in heterogeneous photocatalytic reactors of different scales is proposed. The model is based on the concept of effective radiation [20], in which, the radiant field is quantified as an invariable and isotropic energy flux of photons, which is independent of the propagation direction and is particular suitable for application in solar powered photoreactors.

\section{Methodology}

\subsection{Effective Radiation Model (ERFM) postulates}

The following postulates were made with regard to the effective-radiation concept [20]:

Postulate 1. Considering an isotropic phase-space, which contains suspended particles in a fluid phase, the net global effective radiant power that arrive to a surface $a(\chi)$, identified by a vector of coordinate $\underline{\boldsymbol{x}}$, can be calculated summing up the energy $E=h v$ (with $h$ the Planck constant) of each photon of wavelength $\lambda$, frequency $v$ and propagation direction $\underline{\boldsymbol{\Omega}}$. This net global energy will therefore be independent of the propagation direction and the radiation wavelength (Figure 1 visualizes this approach).

Postulate 2. The net global effective energy associated to a phase-space of suspended solid particles in an heterogeneous system, is assumed to have an isotropic nature. Thus, the optical properties of the suspension can be considered as a global parameter, which is independent of the geometric coordinates [20,44].

Postulate 3. Due to the isotropic nature of the system, the scattering probability identical in each direction. Thus, the system of net effective radiation is modeled exclusively using the isotropic phase function.

Postulate 4. The net energy of particle absorption is quantified through the effective volumetric rate of energy absorption (EVREA), which is equivalent to LVRPA in the traditional approach.

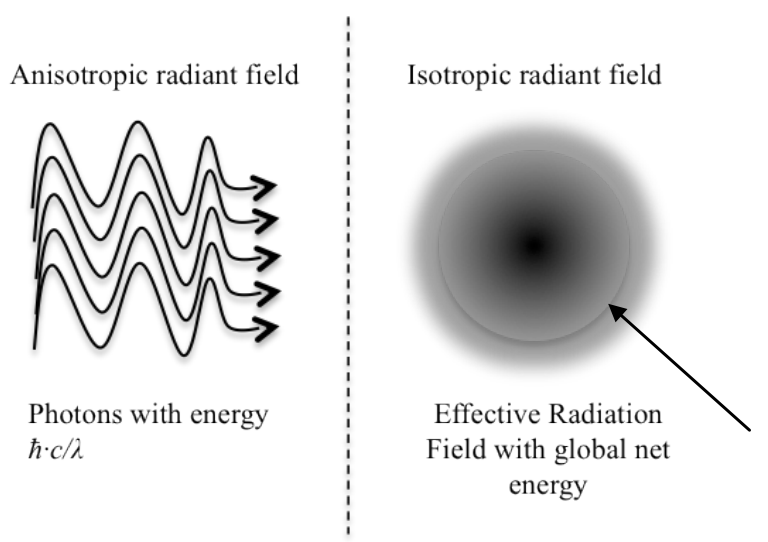

Figure 1. Schematic diagram of the effective radiation concept model.

\subsection{Mathematical Formulation of Effective Radiation Field Model (EFRM)}

The differential radiant energy $d E^{*}$ of a beam ( $\mathrm{J}$ or Ein) in the wavelength interval $d \lambda$ at any time 
$t$ (system at steady-state), which propagates in an unit solid angle $d \underline{\boldsymbol{\Omega}}$, through a surface area $d A$ (see Fig. 2) results in a specific radiation intensity $I_{\lambda}(\underline{\mathbf{x}}, \underline{\boldsymbol{\Omega}}, t)$ equals to:

$I_{\lambda}(\bar{x}, \vec{\Omega})=\lim _{d A, d \delta \dot{d} \cdot d t \cdot d \lambda \rightarrow 0}\left\{\frac{d E^{*}}{d A \cos (\theta) d \Omega d \lambda}\right\}(3)$

Since the system is at steady-state, the differential radiant energy equals to:

$$
d E^{*}=I_{\lambda}(\vec{x}, \vec{\Omega}) d A \cos (\theta) d \Omega d \lambda
$$

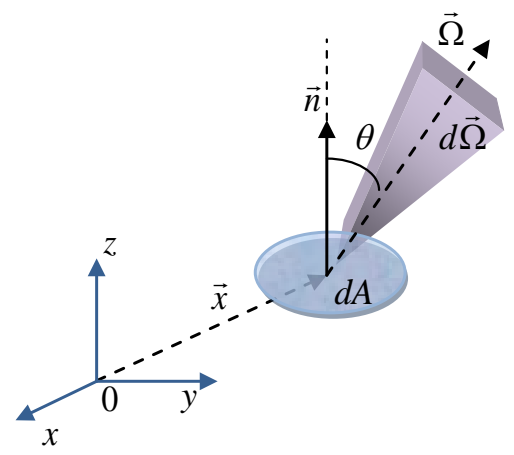

Figure 2. Schematic definition of specific radiation intensity $I_{\lambda}(\underline{x}, \underline{\boldsymbol{\Omega}}, t)[9]$.

As a result of postulate 2, the net radiant power is independent of the direction of propagation of radiation, therefore, it is possible to express the radiation intensity as an integral function in all propagation directions $\underline{\boldsymbol{\Omega}}$, by a differential area ds, as follows:

$$
\begin{aligned}
& d s=d A \cos (\theta) \\
& \int_{\Omega} \frac{d E^{*}}{d s} d \Omega=\int_{\Omega} I_{\lambda}(\vec{x}, \vec{\Omega}) d \Omega d \lambda \\
& \frac{d}{d s} \int_{\Omega} E^{*} d \Omega=\int_{\Omega} I_{\lambda}(\vec{x}, \vec{\Omega}) d \Omega d \lambda
\end{aligned}
$$

Then, $\xi^{E f f}$ is defined as the global, net radiant energy of the photon flux with a wavelength $d \lambda$ through equation (7):

$$
\xi^{E f f}=\int_{\Omega} E^{*} d \Omega
$$

Using the definition of Spectral Incident Radiation $G_{\lambda}(\underline{\mathbf{x}})$ [15]:

$$
G_{\lambda}(\vec{x}, t)=\int_{\Omega} I_{\lambda}(\vec{x}, \vec{\Omega}) d \Omega
$$


Replacing (7) (8) in (6b), the expression for the net differential incident energy on the surface $d s$ of an arbitrary particle $p$ in a heterogeneous system of suspended solid particles is obtained.

$$
d \xi^{E f f}(\vec{x}, \lambda)=G_{\lambda}(\vec{x}, t) d s
$$

Integrating Eq. (9) over the external surface of an arbitrary particle considering that the particles are rigid non-porous spheres with an uniform size and summing for all $N_{v}$ particles in the suspension, it follows:

$$
\xi_{T, \lambda}^{E f f}(\vec{x}, \lambda)=\int_{s} d \xi^{E f f}(\vec{x}, \lambda)=\sum_{k=1}^{N v} \int_{s} G_{\lambda, k}(\vec{x}, t) d s=N_{V} \int_{s} G_{\lambda, k}(\vec{x}, t) d s
$$

Equation (10) represents the total energy of the effective monochromatic spectral incident radiation accumulated in all semiconductor particles in suspension, $\xi_{T}^{E f f}$, which is independent of the direction of propagation of the incident radiation. Due of the isotropy of the system (postulate 3) in the volumetric concentration of the particles in suspension, the net global effective radiant energy that is absorbed by the semiconductor is:

$$
\frac{d \xi_{T, \lambda}^{E f f}(\vec{x}, \lambda)}{d v} \equiv \xi_{N, \lambda}^{E f f}=\kappa_{\lambda} \zeta_{N, \lambda}^{E f f}(\vec{x}, \lambda)
$$

where $\xi_{N, \lambda}^{E f f}$ is the EVREA for a monochromatic radiant energy (energy/volume-time) of wavelength $\lambda, \kappa_{\lambda}$ is the volumetric absorption coefficient of the semiconductor, that is the concentration and wavelength dependent, $v$ is the reaction volume and $\zeta_{N, \lambda}{ }^{E f f}$ is the effective superficial rate of energy absorption (ESREA per area unit).

For solar irradiation (a polychromatic radiation source) Eq. 11 is integrated over the entire wavelengths range of the incident spectrum:

$$
\xi_{N}^{E f f}=\int_{\lambda} \xi_{N, \lambda}^{E f f} d \lambda=\int_{\lambda} \kappa_{\lambda} \zeta_{N, \lambda}^{E f f}(\vec{x}, \lambda) d \lambda
$$

Applying the global, constant, optical parameter approach developed by Mueses et al. for solar radiation [20, 44] equation (12) is finally expressed as follows:

$$
\xi_{N}^{E f f}=\kappa \zeta_{N}^{E f f}(\vec{x})
$$

where $\xi_{N}^{E f f}$ is the net effective volumetric rate of energy absorption $\left(\mathrm{W} / \mathrm{m}^{2}\right)$, see figure $1, \kappa$ is the global volumetric absorption coefficient of the semiconductor, and $\zeta_{N}$ Eff is the net, global effective radiation per area unit, which is solved using a modification of the radiative transfer equation.

\subsection{Modified Radiative Transfer Equation.}

We first consider an arbitrary, differential control volume in a heterogeneous fluid system with 
suspended solid particles $\delta v(\chi)$, where $\chi$ is the space-phase Cartesian position that involves the differential control volume (see Figure 3).
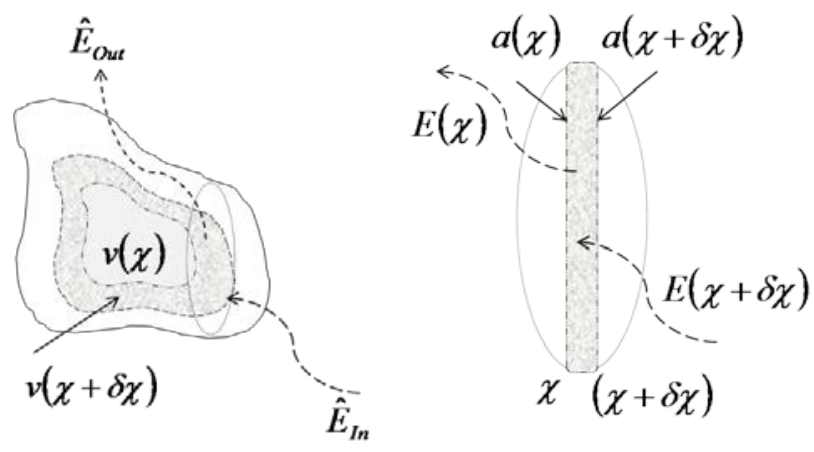

Figure 3. a) Differential control volume, and b) heterogeneous phase-space

The net global effective energy $\zeta_{N}^{E f f}\left(\mathrm{E} \cdot \mathrm{L}^{-2}\right)$ which is incident over the differential area $\delta a(\chi)$, under steady state conditions, involves the following balance for each component:

$\mathrm{E}_{\mathrm{IN}}$, the net radiant-energy flux entering the control volume $\delta v(\chi)$ through $\chi ; \mathrm{E}_{\mathrm{ABS}}$, the energy flux absorbed by the semiconductor; $\mathrm{E}_{\mathrm{S}, \mathrm{OUT}}$, the energy lost by scattering effects; $\mathrm{E}_{\mathrm{S}, \mathrm{IN}}$, the energy gained from the surround by scattering effects; $\mathrm{E}_{\mathrm{OUT}}$, the net flux of radiant energy exiting the control volume $\delta v(\chi)$ through $\chi+\delta \chi$.

Mathematically:

$$
\begin{gathered}
\left.\zeta_{N}^{E f f}(\chi) a(\chi)\right|_{\chi}-\left.\zeta_{N}^{E f f}(\chi) a(\chi)\right|_{\chi+\delta \chi}-[\kappa+\sigma] \zeta_{N}^{E f f}(\chi) \delta v(\chi)+\sigma \varphi_{N}^{E f f}\left(\zeta_{N I}^{E f f}\right)=0 \\
E_{I N}=E_{\text {OUT }} \quad E_{A B S} E_{S, O U T}
\end{gathered}
$$

where $\kappa$ and $\sigma$ are the global volumetric absorption and scattering coefficients of the semiconductor and $\phi_{N}{ }^{E f f}$ is the global phase function of effective scattering which is a function of $\zeta_{N}^{E f f}$.

The new radiative transfer equation modified with the effective radiation approach considers a global net energy flux, which is independent of the direction of propagation. Considering a phase space as a function of an arbitrary coordinate $\chi$, it is given by equation (15):

$$
\frac{1}{a(\chi)} \frac{\partial}{\chi}\left[\zeta_{N}^{E f f}(\chi) a(\chi)\right]=-[\kappa+\sigma] \zeta_{N}^{E f f}(\chi)+\sigma \varphi_{N}^{E f f}\left(\zeta_{N}^{E f f}\right)
$$

where $a(\chi)$ is the perpendicular transfer area to the energy flux $\left(\mathrm{m}^{2}\right)$.

For a plane geometry with constant radiation along the axial direction, $a(\chi)$ is constant, $\chi=z$ and the phase space is confined between $[0, \delta]$, with $\delta$ the reactor thickness. Then, the corresponding radiative transfer equation is: 


$$
\frac{\partial}{z} \zeta_{N}^{E f f}(z)=-[\kappa+\sigma] \zeta_{N}^{E f f}(z)+\sigma \varphi_{N}^{E f f}(z)
$$

With the global boundary condition: $\zeta_{N}^{E f f}=\zeta_{N, 0}{ }^{E f f}$ for $z=0$.

For tubular, concentric or cylindrical reactor systems such as CPCs, where the transfer area of the net, effective energy flux is not constant, $\chi=r$ and the phase space is confined between $[0$, $R$ ], with $R$ the reactor radius. Thus, the corresponding radiative transfer equation is:

$$
\frac{1}{r r} \frac{\partial}{r}\left[r \zeta_{N}^{E f f}(r)\right]=-[\kappa+\sigma] \zeta_{N}^{E f f}(r)+\sigma \varphi_{N}^{E f f}(r)
$$

with the global boundary condition: $\zeta_{N}^{E f f}=\zeta_{N, 0}{ }^{E f f}$ for $r=0$.

\subsection{Estimation of the phase function of the global effective scattering model, $\phi_{N}{ }^{\text {Eff }}$}

The description of the scattering properties of a catalyst suspension requires the knowledge of the scattering coefficient $\sigma$ and the scattering function $\phi_{N}{ }^{E f f}[10,11,36]$. The isotropic phase function is consistent with the concept of effective radiation field model in which the directions of propagation of the photons are not distinguished, and therefore it was considered the most appropriate in this model [20]. A mathematical formulation similar to the modified RTE (Eqs. 14-15) around the phase space in Figure 3 was considered:

$$
\begin{aligned}
& \left.\left.\varphi_{N}^{E f f}(\chi) a(\chi)\right|_{\chi+\delta \chi}-\left.\varphi_{N}^{E f f}(\chi) a(\chi)\right|_{\chi}+\mid \sigma \zeta_{N}^{E f f}(\chi)-\kappa \varphi_{N}^{E f f}(\chi)-\sigma \varphi_{N}^{B f f}(\chi)\right\rfloor v(\chi)=0 \\
& \text { Scattering }_{I N} \quad \text { Scattering }_{O U T} \text { Scattering }_{S, I N} \text { Scattering }_{A B S} \text { Scattering }_{S, O U T}
\end{aligned}
$$

where, Scattering ${ }_{I N}$ is the net global effective energy by scattering entering the phase space, Scattering $_{\text {OuT }}$ is the net global effective energy output, Scattering ${ }_{S, I N}$ is the gain by scattering related to the net global effective energy $\zeta_{N}^{E f} f$ not-absorbed, Scattering ${ }_{A B S}$ is the global, dispersed energy lost by scattering effect. Analogous to the modified RTE (Eq. 15), an equation for radiation scattering is obtained, given by Eq. 19:

$$
\frac{1}{a(\chi)} \frac{\partial}{\chi}\left[\varphi_{N}^{E f f^{\prime}}(\chi) a(\chi)\right]=[\kappa+\sigma] \varphi_{N}^{E f f^{\prime}}(\chi)-\sigma \zeta_{N}^{E f f}(\chi)
$$

The expressions for plane (or axial) geometry and for cylindrical systems are:

$$
\begin{aligned}
& \frac{\partial}{z} \varphi_{N I}^{B f f}(z)=-[\kappa+\sigma] \varphi_{N}^{E f f}(z)+\sigma \zeta_{N}^{E f f}(z) \\
& \frac{1}{r} \frac{\partial}{r}\left[r \varphi_{N}^{E f^{f f}}(r)\right]=-[\kappa+\sigma] \varphi_{N}^{E f f}(r)+\sigma \zeta_{N}^{E f f}(r)
\end{aligned}
$$

\section{Results and discussion}




\subsection{Model solution: Plane-geometry case}

The global isotropic characteristics in the modified radiative transfer equation make use of the characteristic photon path length $\lambda_{0}$ proposed by Özisik (Özisik, 1973) [34, 35, 45], and defined as the inverse of the volumetric coefficient of extinction $\beta$.

$$
\lambda_{0}=\frac{1}{\beta}=\frac{1}{\kappa+\sigma}
$$

Extending this definition to the effective radiation concept, and applying it the to plane geometry for $\zeta_{N}{ }^{E f f}$ and $\phi_{N}{ }^{E f f}$, (Eq. 16 and Ec. 20), then:

$$
\begin{aligned}
& \frac{\partial}{z} \zeta_{N}^{E f f}(z)=\frac{1}{\lambda_{0}}\left[\omega_{E f f} \phi_{N}^{E f f}(z)-\zeta_{N}^{E f f}(z)\right] \\
& \frac{\partial}{z} \phi_{N}^{E f f}(z)=\frac{1}{\lambda_{0}}\left[\phi_{N}^{E f f}(z)-\omega_{E f f} \zeta_{N}^{E f f}(z)\right] \\
& \omega_{E f f}=\frac{\sigma}{\kappa+\sigma}=\sigma \lambda_{0}
\end{aligned}
$$

where $\omega_{E f f}$ is the global scattering albedo for effective radiation. Equations 23 and 24 are a coupled system with two unknowns, restricted by the boundary conditions at the plate ends:

$$
\begin{aligned}
& \text { C.F.1. } \quad \zeta_{N}^{E f f}=\zeta_{N, 0}^{E f f} \quad ; \quad z=0 \\
& \text { C.F.2. } \phi_{N}^{E f f}=0 \quad ; \quad Z=\delta
\end{aligned}
$$

At $z=0$, the value for the net global energy corresponds to the value in the reactor wall exposed to the incident light. Scattering radiation fluxes do not exist for $z=\delta$.

The equation system obtained for plane geometry has a mathematical structure analogous to the Two-Flux Model (TFM) [35] and the Six-Flux Model (SFM) [36] balance equations, hence, it is postulated that the ERFM is a multidimensional extension of radiation scattering $(\mathrm{TFM} \rightarrow \mathrm{SFM} \rightarrow \mathrm{ERFM})$. Nevertheless, it is important to observe that the TFM and the SFM were obtained using a diffusely reflecting phase function, while the ERFM has exclusively isotropic characteristics. The system solution is given as an analogous form to the one proposed by Brucato et al. [35, 36] obtained for TFM and SFM:

$$
\begin{aligned}
& \zeta_{N}^{E f f}=\frac{\zeta_{N, 0}^{E f f}}{(1-\Gamma)} e^{-\frac{z}{\lambda^{E f f}}}\left[1-\Gamma e^{-\frac{z}{\lambda^{E f f}}}\right] \\
& \phi_{N}^{E f f}=\frac{\zeta_{N, 0}^{E f f}}{\lambda^{E f f}(1-\Gamma)}\left[\left(1-\sqrt{1-\omega_{E f f}^{2}}\right) e^{-\frac{z}{\lambda^{E f f}}}-\Gamma\left(1+\sqrt{1-\omega_{E f f}^{2}}\right) e^{-\frac{z}{\lambda^{E f f}}}\right]
\end{aligned}
$$


with

$$
\begin{aligned}
& \Gamma=\frac{1-\sqrt{1-\omega_{E f f}^{2}}}{1+\sqrt{1-\omega_{E f f}^{2}}} e^{-2 \frac{\delta}{\lambda^{E f f}}} \\
& \lambda^{E f f}=\frac{\omega_{E f f}}{\sigma \sqrt{1-\omega_{E f f}^{2}}}
\end{aligned}
$$

Finally, the equation for the evaluation of EVREA using the concept of effective radiation is given by:

$$
\xi_{N}^{E f f}=\frac{\zeta_{N, 0}^{E f f}}{\lambda^{E f f} \omega_{E f f}(1-\Gamma)}\left[\left(\omega_{E f f}-1+\sqrt{1-\omega_{E f f}^{2}}\right) e^{\frac{-z}{\lambda^{E f f}}}+\Gamma\left(\omega_{E f f}-1-\sqrt{1-\omega_{E f f}^{2}}\right) e^{\frac{z}{\lambda^{E f f}}}\right]
$$

The mathematical structure of the Effective Radiation Field Model (ERFM), applies to the plane geometry with constant incident radiation on the plane perpendicular to the flux direction, and to the cylindrical geometry with constant incident radiation along the axial direction and constant transfer area. Thus, it is a one-dimensional anisotropic function in the transfer direction, but isotropic on the incidence area.

\subsection{Simulation of Radiant Field Using ERFM of Heterogeneous Photocatalytic Reactors}

\section{Radiation field simulation}

The optical properties of $\mathrm{TiO}_{2} \mathrm{P} 25$ (Degussa-Evonik) photocatalyst reported by Satuf et al. [46] and Colina-Márquez et al. [25, 26] $\left(\sigma=1297.75 \mathrm{~m}^{2} / \mathrm{Kg} ; \kappa=174.75 \mathrm{~m}^{2} / \mathrm{Kg}\right)$ were considered to simulate and validate the ERFM for the evaluation of heterogeneous radiant fields in photocatalytic reactors. It was validated for an hypothetic flat plate photoreactor of infinite area and of thickness $\delta$, with constant incident light flux $\zeta_{N, 0}$ Eff. The rate of radiant energy absorption was expressed independent of the incident light flux at the reactor transparent window $\left(\xi_{N}^{E f f} /\right.$ $\left.\zeta_{N, 0}{ }^{E f f}\right)$.

Figure 4 shows the EVREA as a function of position at different catalyst concentrations. The ERFM describes the shielding and scattering of light by the suspended catalyst particles. The main findings are:

At high catalyst concentrations $\left(\mathrm{TiO}_{2}>1.0 \mathrm{~g} / \mathrm{L}\right)$ the energy values are maximum in the region near the reactor wall exposed to radiation $(\chi / \delta<0.1)$, but the energy abruptly decrease as the optical thickness increases $[4,7]$.

The interaction of the photons with the catalyst particles in the limit layer region $\underline{(\chi / \delta<0.1)}$ is high since the shielding effects are insignificant, and the scattering effects are at the highest level, substantiating a high activation energy for the catalyst.

As the optical thickness increases (at the same loading of the catalyst) the optical shielding of light overlap the scattering effects and the absorption of the radiant energy radically reduces [8, $9,25,26]$. 
At low catalyst concentration, the scattering of radiant energy is more uniform ( $\mathrm{x} / \delta>0.3)$ across the reactor.

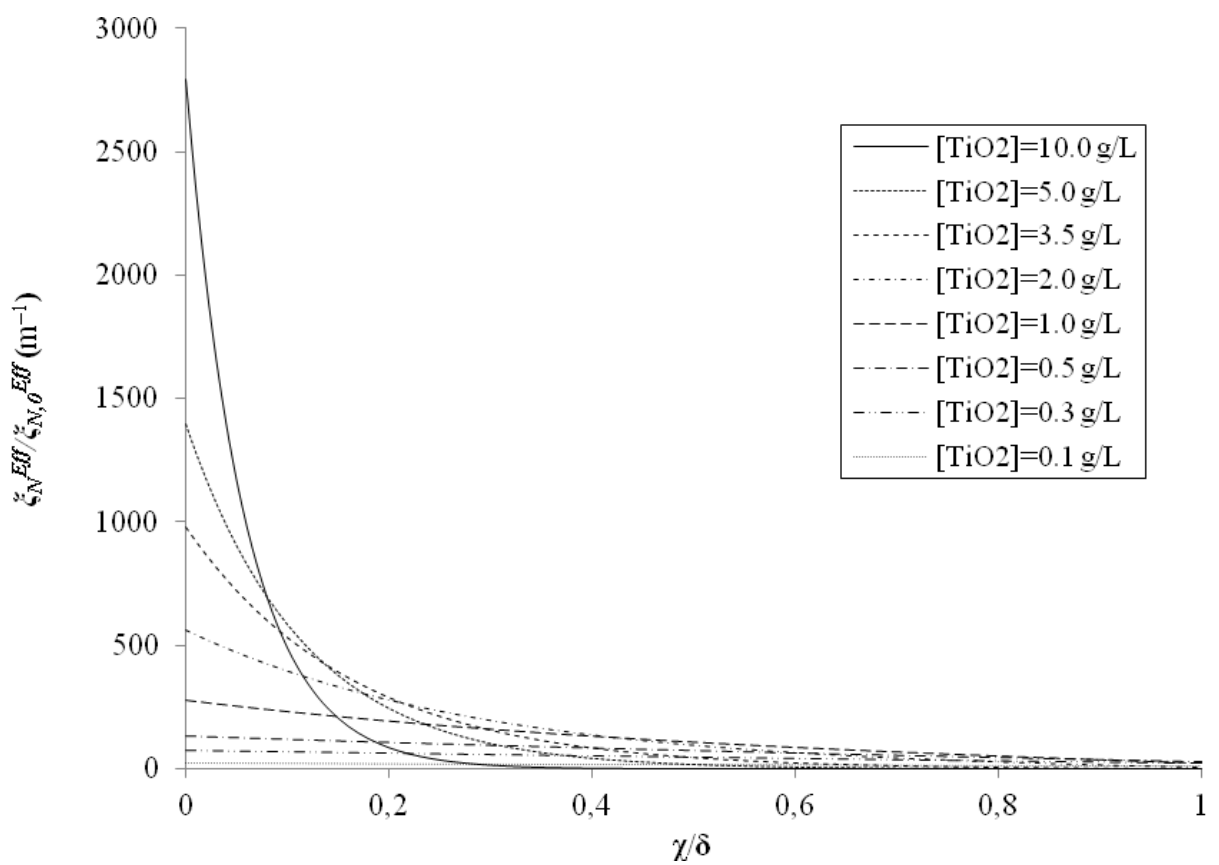

Figure 4. Radiant field simulation for EVREA dimensionless $\xi_{N}{ }^{E f f} / \zeta_{N, 0}$ Eff using ERFM in an infinite area plate and a thickness $\delta=0.020 \mathrm{~m}$. For $\mathrm{TiO}_{2}-\mathrm{P} 25$ Degussa, $\sigma=1297.75 \mathrm{~m}^{2} / \mathrm{Kg} ; \kappa$ $=174.75 \mathrm{~m}^{2} / \mathrm{Kg}$.

Comment [GL1]: Why are the units in the $Y$-axis $(m-1)$ ? Should this be dimensionless?

Figure 5 shows the OVREA as a function of the by apparent optical thickness, with $\tau_{\text {Eff }}$ calculated from Eq. 32 [35]. The definition of Overall Volumetric Rate of Energy Absorption (OVREA) was used (Eq. 33) [20, 44].

$$
\tau_{E f f} \equiv \delta / \lambda^{E f f}
$$

$$
\text { OVREA } \equiv \frac{1}{\delta} \int_{\delta}\left(\frac{\xi_{N}^{E f f}}{\zeta_{N, 0}^{E f f}}\right) d \delta
$$

It is shown that when the optical thickness tends to zero ( $\left.\tau_{\mathrm{Eff}}>0.001\right)$, the OVREA reaches maximum values of energy availability, and it linearly increase with catalyst loading. This results is in agreement with others works $[8,9,25,26]$

At low optical thicknesses (0.01-0.05), the OVREA prediction tends to be asymptotic with the concentration increase (14900 at $0.01 ; 6400$ at 0.025 and 3500 at 0.05$)$, and its values are not above the $10 \%$ of the ones reached to low optic thicknesses and the same loading of catalysts.

Comment [GL2]: I am not sure reference 8 shows what reported in the previous sentence. Please check again with this reference.

Comment [GL3]: Fig 5 results. I dont understand these data. I am missing something. I think it is not correct. Eq. 33 is simply an average across the reactor thickness. So why in Fig. 5 the OVREA reaches such a high values. Please check the physical meaning of the results in Fig. 5 


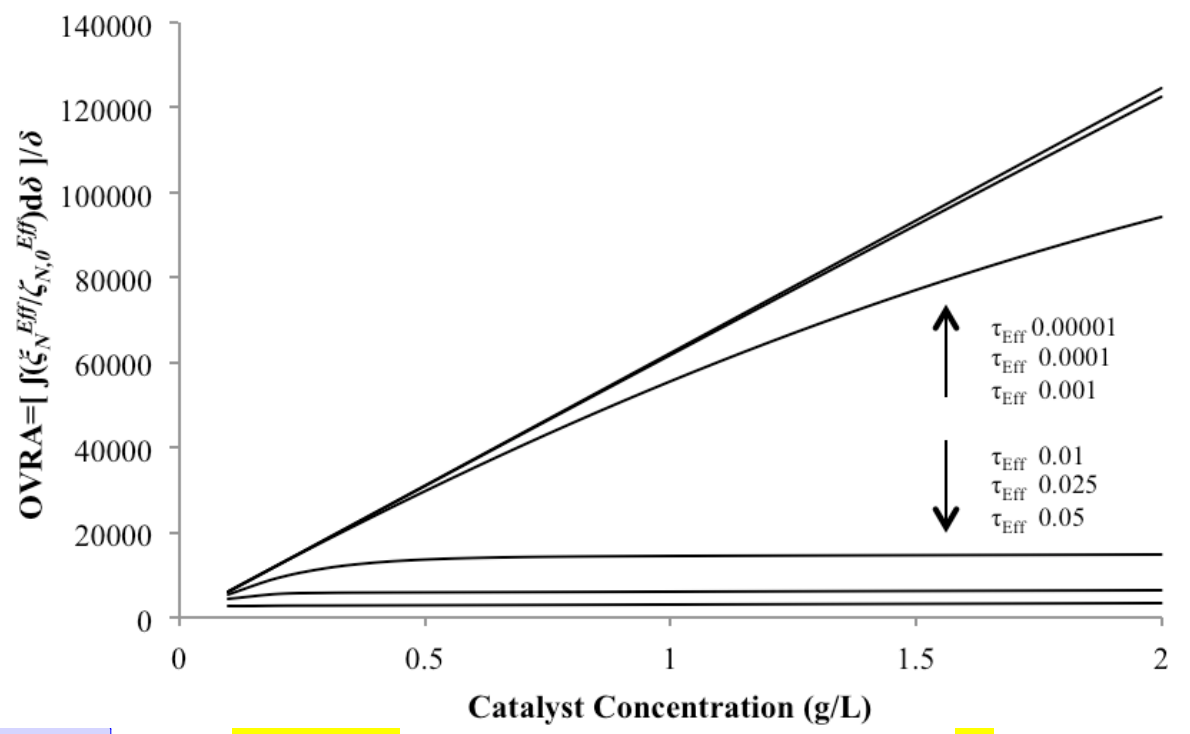

Figure 5. OVREA as a function of catalyst concentration for different optical thicknesses $\tau_{\text {Eff }}$ calculated from the ERFM.

\section{Simulation of scattering effects}

Figure 6 shows the profile of the scattering function $\phi_{\mathrm{N}}{ }^{\text {Eff }}$ as a function of loading catalyst along the slab thickness. Radiation scattering increases linearly from the back wall at low catalyst concentrations, below $0.1 \mathrm{~g} / \mathrm{L}$ (Fig. 6a), while it follows an exponential decrease function at higher catalyst concentrations, above $0.3 \mathrm{~g} / \mathrm{L}$ (Fig. 6b) since at higher catalyst loading the light shielding effect becomes significant. The performance found for this model is physically consistent with literature reports [10-12, 13, 23, 36].

The $\chi / \delta$ coordinate can be used to distinguish two energetic regions. A high energy region (near the reactor front wall) and an area of low energy near the back wall. The amplitude of these areas can be varied by changing the catalyst loading. The optimum for the conditions of this simulations is about $0.3 \mathrm{~g} / \mathrm{L}$.

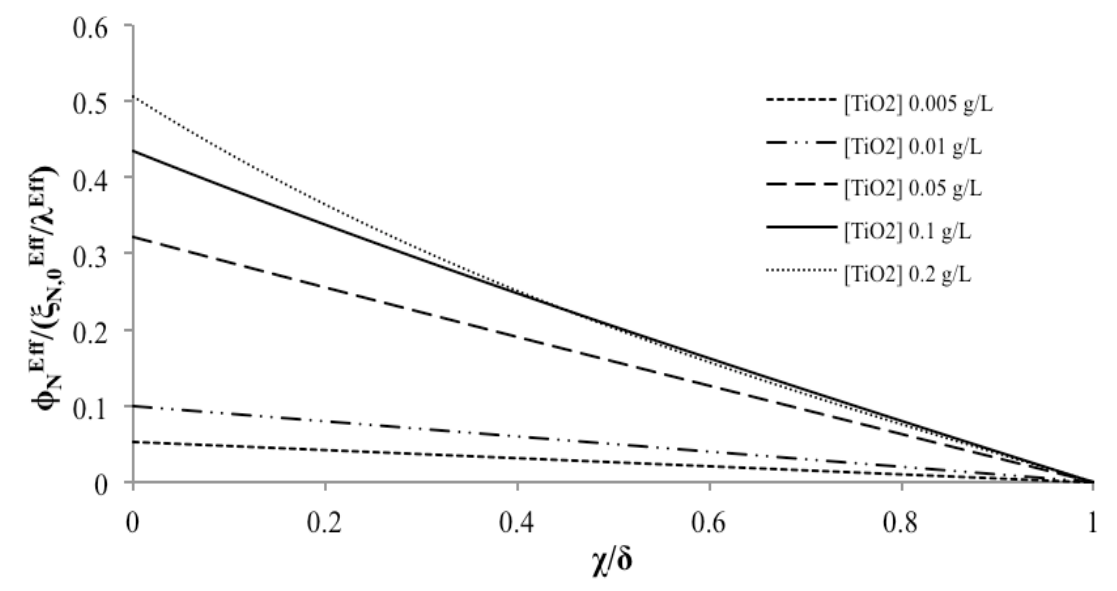




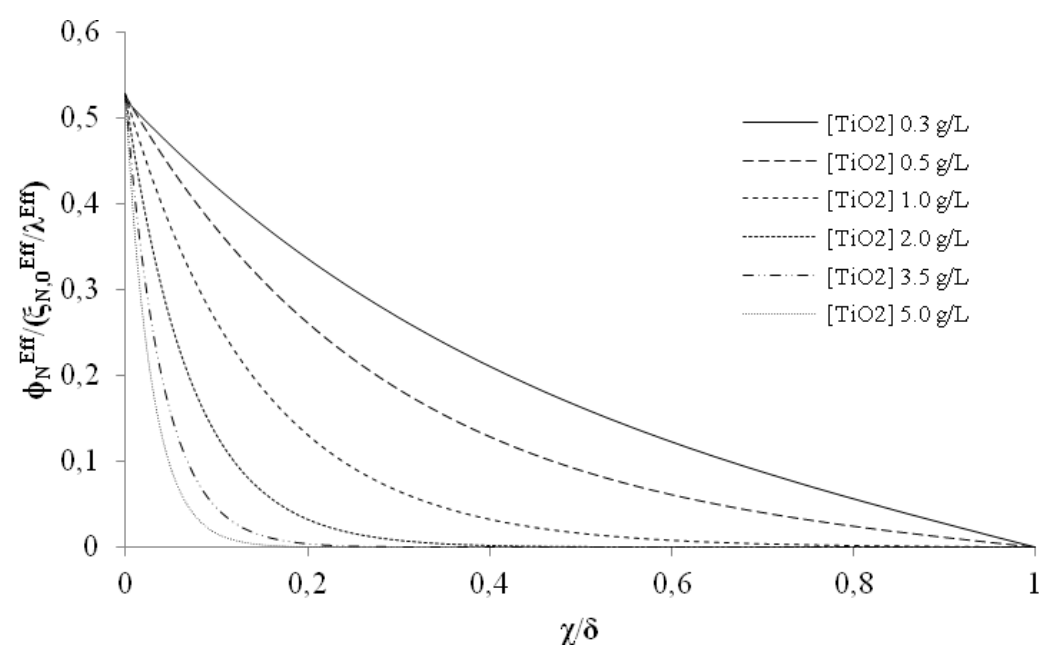

(B)

Figure 6. Simulation of the scattering function $\boldsymbol{\phi}_{N}{ }^{E f f} /\left(\zeta_{N, 0}{ }^{E f f} / \lambda^{E f f}\right)$ in a flat plate of an infinite area and thickness $\delta=0.020 \mathrm{~m}$. $\mathrm{TiO}_{2}-\mathrm{P}-25, \sigma=1297.75 \mathrm{~m}^{2} / \mathrm{Kg} ; \kappa=174.75 \mathrm{~m}^{2} / \mathrm{Kg}$. (A) Low concentrations $\left[\mathrm{TiO}_{2}\right]<0.1 \mathrm{~g} / \mathrm{L}$. (B) high concentrations $\left[\mathrm{TiO}_{2}\right]>0.3 \mathrm{~g} / \mathrm{L}$ ).

\subsection{Comparison of ERFM with SFM}

Figure 7 shows the profiles of the dimensioless OVREA calculated with the ERFM and SFM, at different catalyst loadings and at the same incident radiation flux. The two models show numerical differences which results primarily from the between them, the main difference appears in the correction of the scattering albedo $\omega$. This behavior can be explained by use of the different phase funtion:

For the ERFM with isotropic phase function, the value for scattering albedo is 0.8813 . This value was obtained with integrated values of absorption $\left(\kappa=174.75 \mathrm{~m}^{2} / \mathrm{Kg}\right)$ and specific extinction $\left(\beta=1472.50 \mathrm{~m}^{2} / \mathrm{Kg}\right)$ coefficients reported by Colina-Marquez et al., and Satuf et al., $[25,26,46]$.

For the SFM with diffuse-scattering phase function [36], The value for scattering albedo was of 0.7586 . This value was obtained by matching the SFM model results to the predictions from a Monte Carlo model. 


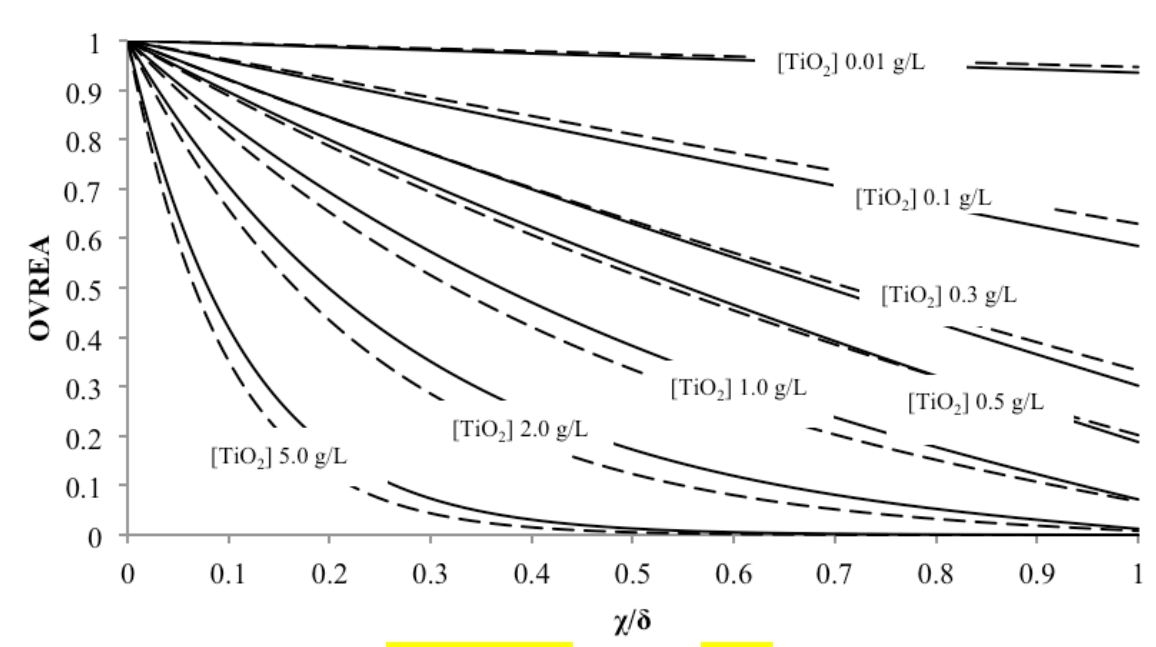

Figure 7. Simulations for the dimensionless OVREA using the ERFM (-) and SFM (---) at different catalyst concentrations.

Moreover, to low catalyst concentrations $\left(\left[\mathrm{TiO}_{2}\right]<0.5 \mathrm{~g} / \mathrm{L}\right)$ the OVREA do not present differences between the models (relative errors less to $2 \%$ referred to SFM), then at this conditions, the difference in the scattering phase function with the two models has little influence on the OVREA profiles.

\subsection{Extending the ERFM model to the cylindrical geometry and validation}

One significant advantage of this new modeling approach is that it can be easily applied for the simulation of radiation fields in photoreactors with fluctuating radiation sources, such as solarscale photocatalytic reactors. Among many reactor configurations, the compound parabolic collector (CPC) is one of the most efficient for application of heterogeneous photocatalysis at solar scale [25, 47-53]. The application of the RTE to this geometry requires a mathematical change to cylindrical coordinates. Nevertheless, due to the mathematical complexity to obtain an analytical solution for these equations, the solution for a plane-geometry was extend to the cylindrical geometry, using the methodology proposed by Li Puma et al. [25, 26, 30-32, 54].

In this approximation, the linear solution of the RTE is extended to equations of bi-dimensional coordinate systems $(r, \theta)$ as a punctual vector in coordinate $\chi$. For this modification to be valid, the thickness $\delta$ becomes variable and corrects the optical thickness as well as the evaluation coordinate of the incident radiant energy $[25,26]$. The modified model is:

$$
\xi_{N}^{E f f}(r, \theta)=\frac{\zeta_{N, 0}^{E f f}}{\lambda^{E f f} \omega_{E f f}(1-\Gamma)}\left[\left(\omega_{E f f}-1+\sqrt{1-\omega_{E f f}^{2}}\right) e^{\frac{-r_{P}(r, \theta)}{\lambda^{E f f}}}+\Gamma\left(\omega_{E f f}-1-\sqrt{1-\omega_{E f f}^{2}}\right) e^{{\frac{r_{P}(r, \theta)^{-}}{\lambda^{E f f}}}^{-}}\right.
$$

with

$$
x_{P}=r \cos (\theta)
$$

$\delta=2 \sqrt{R^{2}-x_{P}^{2}}$
Comment [GL5]: I still believe that such comparison is not so meaningful. In my opinión we need to compare ERFM and SFM using teh same optical parameters. No meangful conclusions can be drawn if the optical parameters used in teh two model are different. I suggest to recalculate the SFM results using omega $=0.8813, \kappa$ $=174.75 \mathrm{~m}^{2} / \mathrm{Kg}$ ) and specific extinction $\left(\beta=1472.50 \mathrm{~m}^{2} / \mathrm{Kg}\right)$.

The discussion in this section needs to be revised in accordance with the new plot.
Comment [GL7]: This sentence needs to be revised. I do not understand what you want to say. 


$$
r_{p}^{\prime}=\delta / 2-r \sin (\theta)
$$

With reference to CPC reactors, the radiant energy re-directed by the reflectors was modeled by coupling the CPC geometry with the Ray Tracing technique $[25,26,55]$. The distance $\delta$ in the reactor tube crossed by each reflected photon light beam is [25, 26]:

$$
\delta=\sqrt{\left(x_{i+1}-x_{i}\right)^{2}+\left(y_{i+1}-y_{i}\right)^{2}}
$$

Finally, both the ERFM and SFM models were evaluated using the cylindrical geometry approximation in a differential tubular reactor with UV lamps. The performance was compared with simulation data of the LVRPA provided by Zalazar and Cassano [6], obtained by the rigorous solution of the RTE using the discrete ordinate method-DOM and assuming an isotropic scattering phase function $[10,12,15]$.

The obtained results (see Figure 8) show that SFM as well as ERFM are far from the RTE solution for DOM at very high optical thicknesses, but they present errors less to $2.5 \%$ in regions close to the lighted wall of the photoreactor.

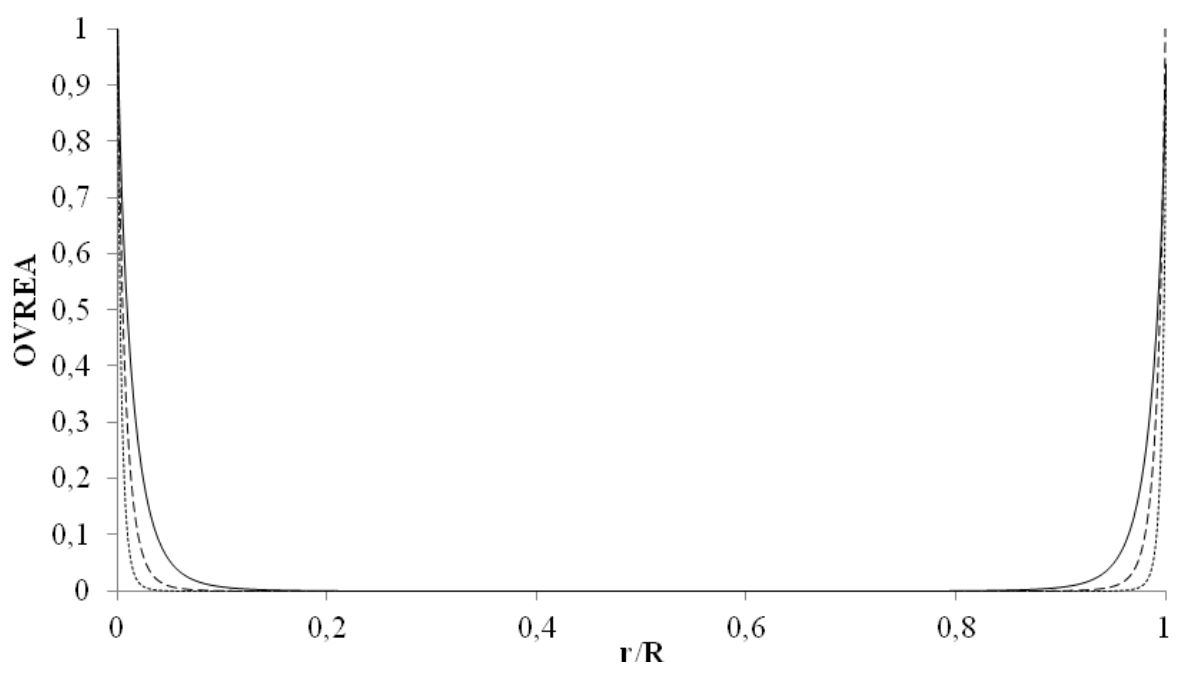

Figure 8. OVREA simulation in a differential tubular photocatalytic reactor with UV lamps for DOM (-) (from Cassano \& Zalazar simulations [12]), ERFM (--) y SFM ( $\cdots$ ). Parameters $\kappa=411.32 \mathrm{~m}^{2} / \mathrm{Kg} ; \beta=3897.4946 \mathrm{~m}^{2} / \mathrm{Kg}$ are to Aldrich- $\mathrm{TiO}_{2}$ semiconductor $\left[\mathrm{TiO}_{2}\right.$ Aldrich $]=1.0 \mathrm{~g} / \mathrm{L} ; \mathrm{L}_{\mathrm{R}}=\mathrm{d}_{\mathrm{R}}=0.052 \mathrm{~m}$.

However, OVREA simulations, when using ERFM, are low with respect to simulations with DOM. This is attributed to the phase function associated to both methods. According to Cassano et al. $[10,12,46]$, the phase function that better represents the radiant field distribution in this type of heterogeneous systems is the isotropic phase function, which was validated by comparing simulations obtained by solving the theoretical equation of Mie function [10, 46].

Mueses et al. (2010) corroborated this result evaluating the phase function change to predict volumetric rates of photon absorption, using the RTE solution with the DOM method to estimate quantum yields in the dichloroacetic acid degradation in a laboratory-scale reactor [23]. 
Quantum yields were compared with a reference value evaluating the LVRPA by solving Mie equation. The same as Alfano et al. 2008 [11] it was found that the isotropic phase function predicts, with discrepancies less to $1 \%$, LVRPA simulations with respect to theoretical solutions and quantum yields of the system $[11,23]$.

The latter results demonstrate that the proposed postulates are valid to formulate the effective radiation field model, ERFM.

\section{Conclusions}

In this study we have presented a new model to estimate the radiation field in heterogeneous photocatalytic reactors. The model is focused on the concept of effective radiation, which considers that radiation distribution into a heterogeneous phase-space can be described as a net global energy flux of incident photons, independent of propagation direction. This postulate permits the model to be considered as an isotropic system with constant global optical properties, and with an isotropic phase function. From these postulates, modified models of the radiative transfer equation and an equivalent for the radiation scattering function were formulated.

The balance equations of radiant energy obtained for a planar system of infinite area and thickness $\delta$ were topologically identical to SFM and TFM equations; therefore, its solution complies with the same mathematical structure.

The major advance in this model, is that it provides an easy to apply method for estimating the radiation field in photoreactors with fluctuating incident photon fluxes, such as solar photoreactors, which do not require time consuming computational methods.

\section{Acknowledgements}

The authors thank Universidad de Cartagena-Colombia, Universidad del Valle-Colombia, Universidad Autónoma de Nuevo León - México and Lourgbourgh University - UK for support this paper. Mueses thanks Colciencias for financing his doctoral studies. Machuca-Martínez and Hernandez-Ramirez thank to CONACYT-México for support at the Programa de Estancias Sabáticas Nacionales, Estancias Sabáticas al Extranjero y Estancias cortas para la consolidación de Grupos de Investigación, Grant 233199. Thanks to Professor Cassano and Dr. Cristina Zalazar by provide the simulation data of DOM-RTE.

\section{References}

[1]. M. Pelaez, N.T. Nolan, S.C. Pillai, M.K. Seery, P. Falaras, A.G. Kontos, P.S.M. Dunlop, J. W.J. Hamilton, J.A. Byrne, K. O'Shea, M.H. Entezari, D.D. Dionysiou, A review on the visible light active titanium dioxide photocatalysts for environmental applications. Applied Catalysis B: Environmental. 125 (2012) 331-349.

[2]. A. Hernández-Ramírez, I. Medina-Ramírez. Photocatalytic Semiconductors, Synthesis, Characterization, and Environmental Applications, Springer, Switzerland, 2015, DOI 10.1007/978-3-319-10999-2.

[3].Y. Lan, Y. Lu, Z. Ren, Mini review on photocatalysis of titanium dioxide nanoparticles and their solar applications. Nano Energy. 2 (2013) 1031-1045.

[4]. C. McCullagh, N. Skillen, M. Adams, P.K. Robertson, Photocatalytic reactors for environmental remediation: A review. Journal of Chemical Tecnology and Biotechnology. 86 (2011) 1002-1017. 
[5].R. Vinu, G. Madras, Renewable energy via photocatalysis. Current Organic Chemistry. 17 (2013) 2538-2558.

[6]. J. Wang, X. Wei, J. Shen, X. Lu, J. Xie, M. Chen, Photocatalytic selective transformation of organics. Progress in Chemistry. 17 (2013) 2516-2537.

[7]. G. Palmisano, V. Augugliaro, M. Pagliaro, L. Palmisano, Photocatalysis: A promising route for 21st century organic chemistry. Chemical Communications. 33 (2007) 3425-3437.

[8]. P.J. Valadés-Pelayo, F. Guayaquil Sosa, B. Serrano, H. de Lasa. Photocatalytic reactor under different external irradiance conditions: Validation of a fully predictive radiation absorption model. Chemical Engineering Science. 126 (2015) 42-54.

[9]. P.J. Valades-Pelayo, J. Moreira, B. Serrano, H. deLasa. Boundary Conditions and phase functions in a Photo-CRECWater-II reactor radiation field. Chemical Engineering Science, 107 (2014) 123-136.

[10]. A. Cassano, O. Alfano, Reaction engineering of suspended solid heterogeneous photocatalytic reactors, Catal. Today, 58 (2000) 167-197.

[11]. O. Alfano, A. Cassano, Photoreactor modeling: Applications to advanced oxidation processes, Int. J. Chem. React. Eng., 6 (2008) 1-20.

[12]. C. Zalazar, R. Romero, C. Martin, A. Cassano, Photocatalytic intrinsic reaction kinetics I: Mineralization of dichloroacetic acid, Chem. Eng. Sci. 60 (2005) 5240-5254.

[13]. G. Imoberdorf, A. Cassano, H. Irazoqui, H. O. Alfano, Optimal design and modeling of annular photocatalytic wall reactor, Catal. Today 129 (2007) 118-126.

[14]. Brandi, R.; Citroni, J.; Alfano, O.; Cassano, A.; Absolute quantum yields in photocatalytic slurry reactor, Chem. Eng. Sci., 58 (2003) 979-985.

[15]. J. Duderstadt, R. Martin, Transport theory, Wiley, New York (1979) 21-422.

[16]. H. Cumminis, A. Levanyuk, Light scattering near phase transitions, North Holland Publishing Company, Netherlands, 1988.

[17]. M. Satuf, R. Brandi, A. Cassano, O. Alfano, Modeling of a flat plate slurry reactor for the photocatalytic degradation of 4-chlorophenol, Int. J. Chem. React. Eng. 5 (2007) A59.

[18]. J. Marugán, R. Van Grieken, A. Cassano, O. Alfano, Intrinsic kinetic modeling with explicit radiation absorption effects of the photocatalytic oxidation of cyanide with $\mathrm{TiO}_{2}$ and silica-supported $\mathrm{TiO}_{2}$ suspensions, Appl. Catal., B 85 (2008) 48-60.

[19]. J. Marugán, R. van Grieken, O. Alfano, A. Cassano, Comparison of empirical and kinetic modeling of the photocatalytic oxidation of cyanide, Int. J. Chem. React. Eng., 5, A89 (2007) 1-9.

[20]. F. Machuca-Martínez, J. Colina-Márquez, M. Mueses, Determination of quantum yield in a heterogeneous photocatalytic system using a fitting-parameters model, J. Adv. Oxid. Technol., 11, (2008) 42-48.

[21]. C. Passalia, O. Alfano, R. Brandi, A methodology for modeling photocatalytic reactors for indoor pollutions control using previously estimated kinetic parameters, J. Hazard. Mater. 211-212 (2011) 357-365.

[22]. R. Brandi, G. Rintoul, O. Alfano, A. Cassano, Photocatalytic reactors reaction kinetics in a flate plate solar simulation, Catal. Today., 76 (2002) 161-175.

[23]. J. Mesa, F. Machuca-Martínez, M. Mueses, Efecto de la dispersión de radiación sobre el rendimiento cuántico en la degradación fotocatalítica de ácido dicloroacético, Inf. Tecnol., 23, (2012) 33-42.

[24]. C. Zalazar, M. Labas, C. Martin, R. Brandi, O. Alfano, A. Cassano, The extended use of actinometry in the interpretation of photochemical reaction engineering data, Chem. Eng. J., 109 (2005) 67-81.

[25]. J. Colina-Márquez, F. Machuca-Martínez, G. Li Puma, Radiation absorption and optimization of solar photocatalytic reactors for environmental applications, Environ. Sci. Technol., 44 (2010) 5112-5120.

[26]. J. Colina-Márquez, J.; F. Machuca-Martínez, G. Li Puma, Photocatalytic 
mineralization of commercial herbicides in a pilot-scale solar CPC reactor: Photoreactor modeling and reaction kinetics constants independent of radiation field, Environ. Sci. Technol., 43 (2009) 8953-8960.

[27]. G. Li Puma, Modeling of thin-film slurry photocatalytic reactors affected by radiation scattering, Environ. Sci. Technol., 37 (2003) 5783-5791.

[28]. G. Li Puma, P. Lock-Yue, Modelling and design of thin-film slurry photocatalytic reactors for water purification, Chem. Eng. Sci. 58, (2003) 2269-2281.

[29]. A. Gora, B. Toepfer, V. Puddu, G. Li Puma, Photocatalytic oxidation of herbicides in single-component and multicomponent systems: Reaction kinetics analysis, Appl. Catal. B-Environ., 65 (2006) 1-10.

[30]. G. Li Puma, J. Khor, A. Brucato, Modeling of an annular photocatalytic reactor for water purification: Oxidation of pesticides, Env. Sci. Technol., 38 (2004) 3737-3745.

[31]. G. Li Puma, A. Brucato, Dimensionless analysis of slurry photocatalytic reactors using a two-flux and six-flux radiation absorption-scattering models, Catal. Today, 122 (2007) 78-90.

[32]. G. Li Puma, B. Toepfer, A. Gora, Photocatalytic oxidation of multicomponent systems of herbicides: Scale-up of laboratory kinetics rate data to plant scale, Catal. Today, 124 (2007) 24-132.

[33]. B. Toepfer, G. Li Puma, A. Gora, Photocatalytic oxidation of multicomponent systems of herbicides: Reaction kinetics analysis with explicit photon absorption effects, Appl. Catal., B 68 (2006) 171-180.

[34]. A. Brucato, L. Rizzuti, Simplified model of radiation fields in heterogeneous photoreactors. 1. Case of zero reflectance, Ind. Eng. Chem. Res., 36 (1997) 47404747.

[35]. A. Brucato, L. Rizzuti, Simplified model of radiation fields in heterogeneous photoreactors. 2. Limiting "Two-Flux" Model for the case of reflectance greater than zero, Ind. Eng. Chem. Res., 36 (1997) 4748-4755.

[36]. A. Brucato, A. Cassano, F. Grisafi, G. Montante, L. Rizzuti, G. Vella, Estimating radiant fields in flat heterogeneous photoreactors by the six-flux model, AIChE J., 52 (2006) 3882-3890.

[37]. W. O’Hirok, C. Gautier, A three-dimensional radiativa transfer model to investigate the solar radiation within a cloudy atmosphere: Part I: Spatial effects, J. Atmos. Sci., 55 (1997) 21-62-2179.

[38]. T. Deutschmann, S. Beirle, U. Frieß, M. Grzegorski, C. Kern, L. Kritten, U. Platt, C. Prados-Román, J. Puksı te, T. Wagner, B. Werner, K. Pfeilsticker, The Monte Carlo atmospheric transfer model McAtim: Introduction and validation of Jacobians and 3D features, J. Quant. Spectrosc. Ra., 112 (2011) 1119-1137.

[39]. J. Petrasch, S. Haussener, W. Lipiński, Discrete vs continuum-scale simulation of radiative transfer in semitransparent two-phase media, J. Quant. Spectrosc. Ra., 112, 9 (2011) 1450-1459.

[40]. C. Passalia, O. Alfano, R. Brandi, Modelling and experimental verification of a corrugated plate photocatalytic reactor using computational fluid dynamics, Ind. Eng. Chem. Res. 50 (2011) 9077-9086.

[41]. F. Trujillo, T. Safinski, A. Adesina, Oxidative photomineralization of dichloroacetic acid in an externally-irradiated rectangular bubble tank reactor: Computational Fluid Dynamics Modeling and Experimental Verification Studies, Ind. Eng. Chem. Res., 49 (2010) 6722-6734.

[42]. F. Jović, V. Kosar, V. Tomašić, Z. Gomzi, Non-ideal flow in an annular photocatalytic reactor, Chem. Eng. Res. Des., 90 (2012) 1297-1306.

[43]. Z. Wang, J. Liu, Y. Dai, W. Dong, S. Zhang, J. Chen, CFD modeling of a UV-LED photocatalytic odor abatement process in a continuous reactor, J. Hazard. Mater. 215-216 (2012) 25-31

[44]. M. Mueses, F. Machuca-Martinez, G. Li Puma, Effective quantum yield and reaction rate model for evaluation of photocatalytic degradation of water 
contaminants in heterogenouos pilot-scale solar photoreactors, Chem. Eng. J.,. 215216 (2013) 937-947.

[45]. M. Özisik, Radiative Transfer and interactions with conduction and convection, Wiley, New York (1973).

[46]. M. Satuf, R. Brandi, A. Cassano, O. Alfano, Experimental method to evaluate the optical properties of aqueous titanium dioxide suspensions, Ind. Eng. Chem. Res., 44 (2005) 6643-6649.

[47]. S. Malato, P. Fernández-Ibañez, M. Maldonado, J. Blanco, W. Gernjak, Decontamination and disinfection of water by solar photocatalysis: Recent overview and trends, Catal. Today, 147 (2009) 1-59.

[48]. S. Malato, J. Blanco, R. Maldonado, P. Fernández, D. Alarcón, M. CollaresPereira, J. Farinha, J. Correia de Oliveira, Engineering of solar photocatalytic collectors. Sol. Energy, 77 (2004) 513-524.

[49]. S. Malato, J. Blanco, A. Campos, J. Cáceres, C. Guillard, J. Herrmann, A. Fernández-Alba, Effect of operating parameters on the testing of a new industrial titania catalyst at solar pilot plant scale, Appl. Catal. B-Environ., 42 (2003) 349-357.

[50]. S. Malato, J. Blanco, A. Vidal, C. Richter, Photocatalysis with solar energy at a pilot-plant scale: An overview, Appl. Catal. B-Environ., 37 (2002) 1-15.

[51]. J. Colina-Márquez, D. Díaz, A. Rendón, A. López-Vásquez, F. MachucaMartínez, Photocatalytic treatment of a dye polluted industrial effluent with a solar pilot-scale CPC reactor, J. Adv. Oxid. Technol., 12 (2009) 93-99.

[52]. E. Bandala, C. Estrada, Comparison of solar collection geometries for application to photocatalytic degradation of organic contaminants, J. Sol. Energy Eng. 129 (2007) 22-26.

[53]. E. Bandala, C. Arancibia-Bulnes, S. Orozco, C. Estrada, Solar photoreactors comparison based on oxalic acid photocatalytic degradation, Sol. Energy, 77 (2004) 503-512.

[54]. G. Li Puma, V. Puddu, H. Tsang, A. Gora, B. Toepfer, Photocatalytic oxidation of multicomponent mixtures of estrogens (estrone (E1), 17 $\beta$-estradiol (E2), $17 \alpha$-ethynylestradiol (EE2) and estriol (E3)) under UVA and UVC radiation: Photon absorption, quantum yields and rate constants independent of photon absorption, Appl. Catal. B-Environ., 99 (2010) 388-397.

[55]. J. Colina-Márquez, A. López-Vásquez, F. Machuca-Martínez, Modeling of direct solar radiation in a compound parabolic collector (CPC) with the ray tracing technique, Dyna, 77 (2010) 132-140. 